\title{
Hidden from Plain Sight: Residents' Domestic Violence Screening Attitudes and Reported Practices
}

\author{
Arshiya Baig, MD, MPH, ${ }^{7}$ Elizabeth Shadigian, $M D^{2}{ }^{2}$ Michele Heisler, $M D, M P A^{3,4}$ \\ 'Robert Wood Johnson Clinical Scholars Program, Division of General Internal Medicine and Health Services Research, Department of \\ Medicine, University of California, Los Angeles, CA, USA; '2Department of Obstetrics and Gynecology, University of Michigan School of \\ Medicine, Ann Arbor, MI, USA; ${ }^{3}$ Veterans Affairs Center for Practice Management \& Outcomes Research, VA Ann Arbor Healthcare \\ System, Ann Arbor, MI, USA; ${ }^{4}$ Department of Internal Medicine, Michigan Diabetes Research and Training Center, University of Michigan \\ School of Medicine, Ann Arbor, MI, USA.
}

\begin{abstract}
BACKGROUND: Domestic violence (DV) is prevalent across all racial and socioeconomic classes in the United States. Little is known about whether physicians differentially screen based on a patient's race or socioeconomic status (SES) or about resident physician screening attitudes and practices.
\end{abstract}

OBJECTIVE: To assess the importance of patient race and SES and resident and clinical characteristics in resident physician DV screening practices.

DESIGN, PARTICIPANTS: One-hundred and sixty-seven of 309 (response rate: 54\%) residents from 6 specialties at a large academic medical center responded to a randomly assigned online survey that included 1 of 4 clinical vignettes and questions on attitudes and practices regarding DV screening.

MEASUREMENTS: We measured patient, resident, and clinical practice characteristics and used bivariate and multivariate methods to assess their association with the importance residents place on DV screening and if they would definitely screen for DV in the clinical vignette.

RESULTS: Residents screened the African-American and the Caucasian woman (51\% vs $57 \%, P=.40$ ) and the woman of low SES and high SES (49\% vs $58 \%, P=.26$ ) at similar rates. Thirty-seven percent of residents incorrectly reported rates of DV are higher among African Americans than Caucasians, and 66\% incorrectly reported rates are higher among women of lower than of higher SES. In multivariate analyses, residents who knew where to refer DV victims (adjusted odds ratio $[\mathrm{AOR}]=3.54,95 \%$ confidence interval $[\mathrm{CI}]: 1.43$ to 8.73 ) and whose mentors advised them to screen ( $\mathrm{AOR}=3.46,95 \% \mathrm{CI}: 1.42$ to 8.42 ) were more likely to screen for DV.

CONCLUSION: Although residents have incorrect knowledge about the epidemiology of DV, they showed no racial or SES preferences in screening for DV. Improvement of mentoring and educating residents about referral resources may be promising strategies to increase resident DV screening.

KEY WORDS: domestic violence; domestic violence screening; resident education.

DOI: $10.1111 /$ j.1525-1497.2006.00494.x

J GEN INTERN MED 2006; 21:949-954.

$\mathrm{D}$ omestic violence (DV) is defined as physical, sexual, or psychological harm by a current or former partner or spouse, occurring across all social, economic, and cultural groups. ${ }^{1,2}$ Thirty-one percent of U.S. women report abuse in

Address correspondence and requests for reprints to Dr. Baig: UCLA Clinical Scholars Program, Division of GIM/HSR, Department of Medicine, 911 Broxton Avenue, Third Floor, Los Angeles, CA 90024 (e-mail: abaig@mednet.ucla.edu). their lifetime. ${ }^{3-5}$ Although some studies report low-income and minority women are at higher risk for victimization, racial, and economic disparities are negligible when other factors are taken into account. ${ }^{6-9}$ More than 2 million women are injured by DV, and 500,000 require medical treatment annually. ${ }^{5,10,11}$ Physical health sequelae include a $50 \%$ to $70 \%$ increase in gynecological and central nervous system complications and long-term psychiatric problems. ${ }^{9,12-15}$

Physicians are important in identifying and treating DV victims as many abused women seek medical care after victimization. ${ }^{5,16-19}$ Unfortunately, only $10 \%$ of primary care physicians routinely screening for $\mathrm{DV}^{20,21}$ Provider-related barriers to DV screening include lack of education, lack of time, lack of effective interventions, and fear of offending the patient. $^{20-22}$ Although racial disparities exist in the diagnosis and treatment of many medical conditions, no studies have assessed physician racial and socioeconomic preferences in DV screening. ${ }^{23,24}$

In this study, we assess patient and resident characteristics that influence residents' attitudes and reported practices toward DV screening. We surveyed residents in 6 specialties to: (1) determine whether residents preferentially screen minorities or women of low socioeconomic status (SES); and (2) assess which resident and clinical practice characteristics are associated with screening.

\section{METHODS}

We incorporated reported barriers to DV screening into a 22item online survey to assess residents' knowledge, attitudes, and practices. ${ }^{17-22}$ The survey is published online as an appendix. Questions regarding DV were embedded among a range of preventive screening areas, such as diet, safety belt usage, sexual behavior, and exercise. ${ }^{25}$ The survey included multiple-choice questions and 1 of 4 clinical vignettes that varied by race (African American or Caucasian) and by SES (lawyer or fast food worker) (see Fig. 1). The survey also included 2 open-ended questions regarding when residents screened and what prevented DV screening. Completion time was approximately 10 minutes. The surveys received institutional review board approval. Resident consent was implied by their returning the completed survey. Respondents were compensated with cafeteria gift certificates worth $\$ 1.25$ after survey completion.

Between September and December, 2004, residents across 6 residency programs-Internal Medicine, Obstetrics/

Manuscript received September 27, 2005

Initial editorial decision November 17, 2005

Final acceptance March 23, 2006 
Mrs. J. is a 32-year old [African-

American/Caucasian] married woman who

works as a [cashier at a fast food

restaurant/law professor at the university].

She is coming in today for her annual check-

up. She brings up no concerns today except

for a mild headache and stomach 'pains' that

she's had for several years. She denies any

change in her symptoms. She denies any

fevers, chills, photophobia, neck rigidity,

nausea, vomiting, melena, BRBPR, or

change in her bowel habits. She's had a good

appetite. Her physical examination is

unremarkable. Pap smear was done. She was

also given a refill on her birth control pills.

FIGURE 1. Clinical vignette.

Gynecology (OB/GYN), Pediatrics, Family Medicine, Emergency Medicine, and Combined Medicine/Pediatrics (Med/Peds)— at a single academic medical center, received an electronically mailed link to the online survey, "Resident Attitudes in Primary Care Screening." The administrator preassigned a survey to the residents before sending the soliciting e-mail. Residents were categorized by specialty, divided into 4 groups by numeric order of employee number, and then randomly assigned 1 of the 4 surveys. Nonrespondents received 8 electronically mailed reminders.

\section{Outcome Measures}

To assess the importance of the routine screening areas for each case, respondents were asked, "How important are the following routine screens in this patient?" Respondents chose from 5 responses: very important, somewhat important, neutral, low importance, or not important. To assess intention to screen, residents were asked, "Which of the following (of the routine screening areas during this visit) would you definitely ask about during this visit?" Respondents chose from 4 responses: "would definitely check/ask at this visit," "would check/ask if there was time," "would defer to future visit," or "would not check/ask."

To assess resident knowledge of the prevalence of DV by race and SES, we asked: "Are rates of DV higher among African-American women in the United States than among Caucasian women?" and "Are rates of DV higher among women of lower SES in the United States than among higher SES women?"

\section{Independent Variables}

Based on literature review and discussions with experts, we included resident and practice characteristics found to be associated with physician screening attitudes and practices as independent variables. ${ }^{19-22}$

Patient Characteristics in Clinical Vignette. To assess if residents preferentially screen based on race or SES, we presented 4 cases. We constructed variables for patient race (African American vs Caucasian), SES (high vs low), and combination variables (high/African American, high/Caucasian, low/African American, and low/Caucasian).

Resident Characteristics. Respondents reported their gender, minority status (nonminority vs minority), specialty, first or second half of residency, personal history of DV or sexual abuse, knowing a victim/perpetrator of DV, having cared for victims of DV, and DV training in medical school/residency. Residents were asked 2 open-ended questions regarding when they screened and what prevented screening.

Clinical Practice Variables. To assess clinical practice characteristics, residents were asked if they knew where to refer victims, if they were aware of a clinic protocol for victims of DV, and if their clinic mentor advised screening.

\section{Analyses}

We examined descriptive statistics of the residents' characteristics, attitudes and behaviors, and knowledge of the prevalence of DV. Chi-square analyses were performed to assess differences in perceived importance of screening and intention to screen based on patients' race and SES. Bivariate and multivariate logistic regression analyses assessed the association between resident and clinical characteristics and resident DV screening attitudes and practices. In the multivariate regressions, we included variables that were significantly associated with either of the 2 principal outcomes at a $P<.10$ level in the bivariate analyses. We also evaluated residents' responses to the open-ended questions about barriers they faced to screening.

\section{RESULTS}

A total of 167 of 309 current residents (54\%) from 6 specialties responded to the survey (Table 1). Seventy-four percent of family medicine, $57 \%$ of pediatrics, $59 \%$ of OB/GYN, $47 \%$ of Med/Peds, $46 \%$ of emergency medicine, and $43 \%$ of internal medicine responded. Demographic information about nonresponders was not made available for comparison.

\section{Importance of DV Screening}

One hundred and eight residents thought DV screening was "very important," 51 "somewhat important," 5 neutral, 1 "low importance," and 0 "not important." Because of the positively skewed responses, we classified responses as either "very important" if the respondent ranked screening as "very important" or "not very important" if they chose another response.

\section{Intention to Screen for DV}

Eighty-eight residents "would definitely check," 57 "would check if time allowed," 13 "would defer to a future visit," and 6 "would not check." Because of the positively skewed responses, we classified responses as "would definitely ask" if the respondent "would definitely ask/check" or as "would not definitely ask" if they chose another response.

\section{Patient Characteristics}

Residents placed equal importance on screening the African American and the Caucasian woman $\left(64 \%\right.$ vs $67 \%, \chi^{2}=0.18$, $P=.67)$ and the low and the high-SES woman (63\% vs $68 \%$, $\chi^{2}=0.58, P=.45$ ). Residents were equally likely to definitely screen the African-American and the Caucasian woman (51\% 
Table 1. Characteristics of Study Participants $(N=167)$

\begin{tabular}{lc}
\hline \hline Resident-Physician Characteristics & $N(\%)^{*}$ \\
Field & $69(42)$ \\
Internal medicine & $13(8)$ \\
OB/GYN & $21(13)$ \\
Pediatrics & $26(16)$ \\
Emergency medicine & $15(9)$ \\
Family medicine & $22(13)$ \\
Medicine/pediatrics & $99(60)$ \\
Female & $34(20)$ \\
\%-Minority & \\
Year in residency & $87(52)$ \\
First half & $80(48)$ \\
Second half & \\
Victim of sexual abuse or DV & $16(10)$ \\
Yes or prefer not to say & \\
Known a victim/perpetrator of DV & $88(54)$ \\
Yes or prefer not to say & $146(87)$ \\
Received DV training in medical school & $53(32)$ \\
Received DV training in residency & $129(77)$ \\
Taken care of a DV victim & $80(48)$ \\
Preceptor advises to screen for DV & $108(65)$ \\
Know where to refer DV victims & \\
Have protocol in clinic for DV victims & $26(16)$ \\
Yes & $21(13)$ \\
No & $120(72)$ \\
Do not know & \\
Frequency of screening for DV & $35(21)$ \\
Always & $91(55)$ \\
When red flags present & $36(22)$ \\
Sometimes & $5(3)$ \\
Never & 100 \\
\hline Because of roun & \\
\hline
\end{tabular}

${ }^{*}$ Because of rounding, percentages may not equal 100.

OB/GYN, Obstetrics/Gynecology; DV, domestic violence.

vs $57 \%, \chi^{2}=0.71, P=.40$ ) and the low and the high-SES woman ( $49 \%$ vs $\left.58 \%, \chi^{2}=1.28, P=.26\right)$.

\section{Resident Characteristics}

The majority of residents would only screen for DV if red flags were present. Most residents received training in medical school for DV screening. Only one-third reported receiving residency training. Nine percent of internal medicine, $23 \%$ of Med/Peds, $73 \%$ of emergency medicine, $60 \%$ of family medicine, $46 \%$ of $\mathrm{OB} / \mathrm{GYN}$, and $38 \%$ of pediatrics residents received training during residency.

\section{Clinical Practice Characteristics}

More than one-third of the residents did not know where to refer DV victims. The majority of residents did not have or did not know the clinic protocol for taking care of DV victims. Less than half of the respondents' reported that clinic preceptors advised them to screen for DV.

Clinical practice characteristics differed across specialties. Eighty-seven percent of family medicine, 58\% of emergency medicine, $54 \%$ of OB/GYN, $43 \%$ of pediatric, and $42 \%$ of internal medicine, and $36 \%$ of Med/Peds residents received advice from their preceptor to screen for DV. All family medicine residents and $92 \%$ of emergency medicine residents knew where to refer victims of DV. Forty-eight percent of internal medicine, $68 \%$ of Med/Peds, $57 \%$ of pediatrics, and $69 \%$ of OB/GYN residents knew where to refer victims. Sixty-two percent of emergency medicine residents knew of a protocol for DV victims, compared with $31 \%$ of OB/GYN, $20 \%$ of family medicine, $5 \%$ of pediatric, and $1 \%$ of internal medicine, and $5 \%$ of Med/Peds residents.

\section{Bivariate and Multivariate Analyses}

Table 2 shows the bivariate and adjusted odds ratio of ranking DV screening as being very important and of definitely screening for DV in the vignette. In the bivariate analyses, emergency medicine residents were less likely to state DV screening was important than OB/GYN residents. Residents who had taken care of a DV victim were more likely to report that DV screening was very important than those that thought they had never taken care of a DV victim. In the multivariate logistic regressions, emergency medicine residents remained less likely to report DV screening as being important than OB/GYN residents.

In the unadjusted odds, female residents were more likely to definitely screen for DV than male residents. In comparison with OB/GYN residents, emergency medicine residents and internal medicine residents were less likely to definitely screen. Residents who received DV training in residency, whose preceptors advised them to screen, who knew where to refer victims, who had taken care of a DV patient, and were in their second half of residency were more likely to definitely screen.

In the multivariate analyses, emergency medicine residents and family medicine residents were less likely to screen than OB/GYN residents. Residents whose preceptors advised to screen and who knew where to refer victims were more likely to definitely screen.

\section{Resident Knowledge of Epidemiology of DV in United States}

Domestic violence against women is as prevalent among Caucasians as among African Americans and is equally frequent among high and low SES. ${ }^{8,9}$ Thirty-seven percent of residents incorrectly believed that DV was more prevalent among African-American than Caucasian women, and $66 \%$ of residents falsely believed that DV was more prevalent among women of lower SES.

\section{Reported Barriers to Screening}

Lack of time $(36 \%)$, no $(4 \%)$ or poor $(16 \%)$ training in DV screening, lack of an effective intervention for victims (9\%), risk of offending the patient (11\%), personal discomfort (10\%), and forgetting $(2 \%)$ were noted as barriers.

Other barriers included: 6 emergency medicine residents who stated the triage nurse usually screens for DV before the physician sees the patient, 3 pediatrics residents who stated the parents were always in the room with the patient, 1 pediatrician who stated DV screening was not completely applicable in pediatrics, and $1 \mathrm{OB} / \mathrm{GYN}$ resident and 1 family practice resident who stated the partner was in the room or there was a lack of privacy. Others residents identified barriers such as: "I do not have a relationship with that person if I'm seeing them just once in urgent care," "I'd screen if the patient were pregnant," "I see a minimal number of women at the Veterans Administration Hospital," and "There is no conclusive evidence for benefits from screening," in reference to current USPSTF guidelines. ${ }^{26}$ 
Table 2. Bivariate and Adjusted Odds of Reporting Domestic Violence Screening as Very Important and of Definitely Screening ( $N=167)$

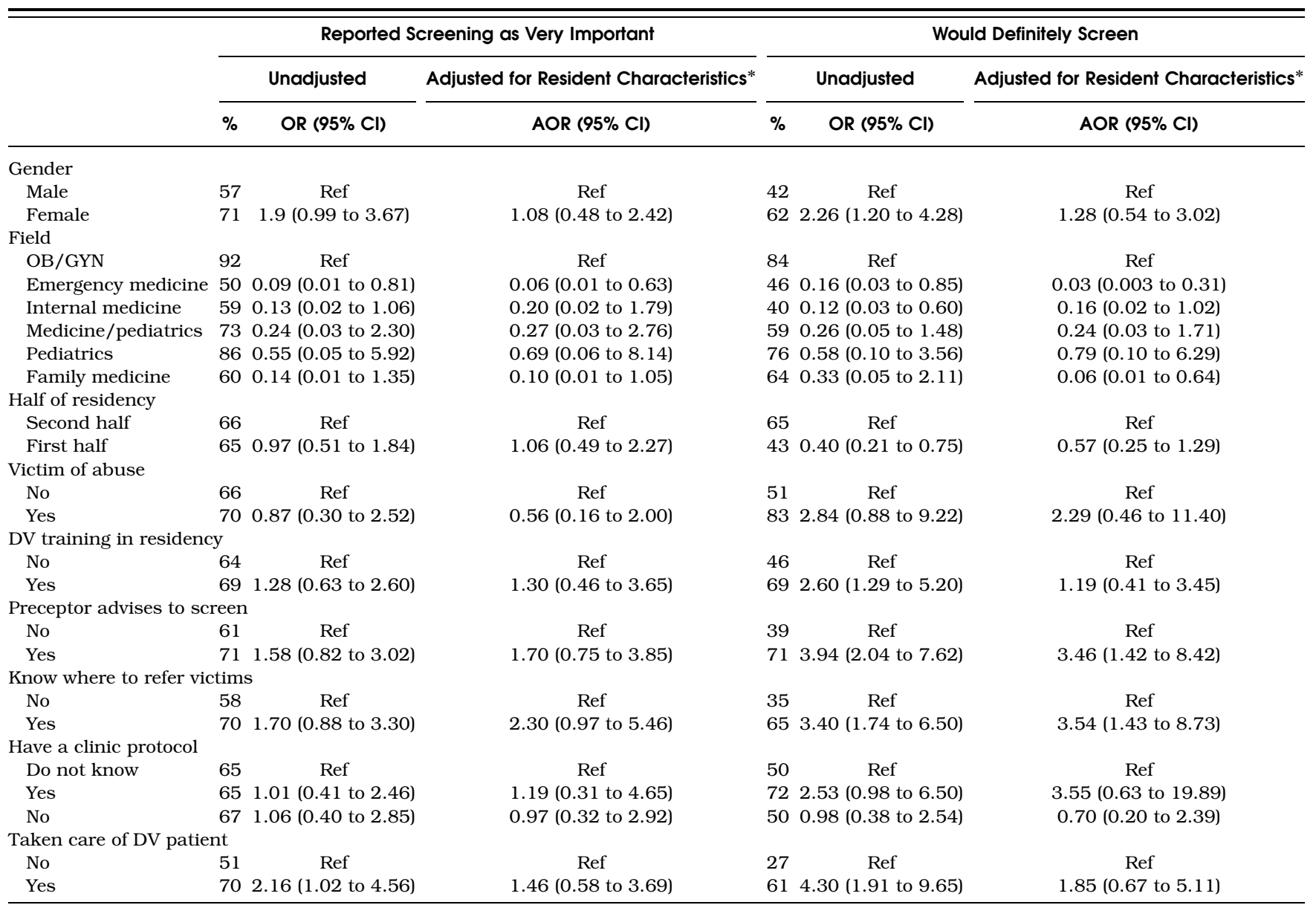

${ }^{*}$ Knowing a victim/perpetrator of DV, minority status of the respondent, and receiving DV training in medical school were not associated with definitely screening for DV in the bivariate analyses. These variables were not adjusted for in the multivariable logistic models.

OB/GYN, Obstetrics/Gynecology; DV, domestic violence; AOR, adjusted odds ratio; OR, odds ratio; CI, confidence interval.

\section{DISCUSSION}

Physicians' a priori assumptions based on inaccurate epidemiological data or social stereotypes can lead to screening and differential treatment of patients by race or class. ${ }^{27,28} \mathrm{Al}-$ though $37 \%$ of residents incorrectly reported that DV was more prevalent among African-American than Caucasian women, and $66 \%$ of residents incorrectly reported higher rates of DV among lower SES women, residents showed no racial or SES preferences in DV screening.

In the multivariate analyses, several clinical practice-but not resident-characteristics were independently associated with whether the resident would definitely screen the patient for DV. Having a preceptor who advises to screen for DV and knowing where to refer victims were the only characteristics independently associated with definitely screening for DV, reinforcing the importance of mentorship in medical education and suggesting that providers are more inclined to ask about problems for which they have solutions. ${ }^{29,30}$ Current educational approaches need reassessment, based on our findings, as many have not shown sustained changes in attitudes and actual rates of DV screening in clinical practice. ${ }^{31-33}$

Prior studies that have evaluated physician characteristics associated with screening have had mixed results. ${ }^{20,21,34}$
We found that resident characteristics, such as gender, year in residency, and personal history of abuse were not independently associated with importance placed on screening or on stated intention to screen for DV. However, emergency medicine residents and family medicine residents were less likely to definitely screen for DV compared with OB/GYNs. These findings are consistent with previous studies that found low rates of DV screening across various specialties. ${ }^{20,21,35}$ The low screening rate among emergency medicine residents may be explained in part by the belief that patients are adequately screened in triage. Unfortunately, many women will only disclose a history of DV when asked by a physician. ${ }^{36}$

Although rates of DV among adult women are very high, men, the elderly, and adolescents are also victims of DV. ${ }^{3,13,37}$ Some pediatric residents did not screen because of privacy concerns and thus may miss DV among their adolescent patients. Other residents did not screen for DV at predominately male VA hospitals, possibly missing DV among this patient population. Further research needs to be conducted to design appropriate interventions to identify male victims of DV.

Residents reported lack of time, personal discomfort, and forgetting to ask, as personal barriers to DV screening, similar to findings in previous studies. ${ }^{20}$ Residents also stated they feared offending the patient, although research suggest that 
patients do not mind being asked. ${ }^{38}$ Although residents stated no or poor training in DV as a barrier, training in residency was not independently associated with priority placed on screening nor on stated intention to screen. There is a need for future research to understand how DV is addressed in the curricula of each residency program.

\section{Limitations}

Our study findings should be interpreted in the face of several limitations. Although our survey included residents from a range of different specialties, our total sample size was small, limiting our power to detect differences in variables for which we found no significant differences. Our sample also came from a single, tertiary care, academic institution and may not be generalizable to other institutions. It is also not possible to assess the influence of organizational factors and of the individual residency programs on the results. The hypothetical cases in the survey may not be representative of actual clinical practice. All the survey responses were self-report and may not correctly represent true behaviors. Although we embedded questions about DV in a range of preventive screening questions, those most intrinsically interested in the subject matter may have been disproportionately likely to participate. Similarly, our response rate of $54 \%$ is comparable with other physician surveys, but residents interested in primary care screening may have been more likely to participate, as evidenced by the nearly double participation rate from family medicine residents compared with other groups. ${ }^{39}$

\section{CONCLUSION}

Many residents incorrectly reported that the prevalence of DV is higher among women of lower SES and who are African American. However, this study did not provide evidence for differential screening. The importance of resident mentorship and provider knowledge of referral sources for DV screening is reinforced by our study. Efforts to improve mentoring and awareness of available resources may be especially promising strategies to increase physician DV screening during residency.

This work was supported by the Department of Internal Medicine residency program at the University of Michigan Health System, the Robert Wood Johnson Foundation's Clinical Scholar Program, and the Department of Veterans Affairs (VA) Health Services Research \& Development (HSR\&D) Service. Dr. Heisler is a VA Research Career Development awardee. The authors would like to thank Mary Bernier at the University of Michigan School of Medicine for managing the online survey and the Internal Medicine Residency Program at the University of Michigan for their encouragement and support of this project. We also appreciate the assistance of Dr. Robert Bjork and the first year Robert Wood Johnson Clinical Scholars at the University of California, Los Angeles in editing this manuscript.

\section{REFERENCES}

1. Saltzman LE, Fanslow JL, McMahon PM, Shelly GA. Intimate Partner Violence Surveillance: Uniform Definitions and Recommended Data Elements. Atlanta, GA: National Center for Injury Prevention and Control, Centers for Disease Control and Prevention; 1999.

2. Heise L, Garcia-Moreno C. Violence by intimate partners. In: Krug EG, Dahlberg LL, Mercy JA, Zwi AB, Lozano R, eds. World Report on Violence and Health. Geneva: World Health Organization; 2002: 87-121.
3. Dahlberg LL, Krug EG. Violence-a global health problem. In: Krug EG, Dahlberg LL, Mercy JA, Zwi AB, Lozano R, eds. World Report on Violence and Health. Geneva: World Health Organization; 2002:1-21.

4. Collins KS, Schoen C, Joseph S, et al. Health Concerns Across a Woman's Lifespan: The Commonwealth Fund 1998 Survey of Women's Health. New York: Commonwealth Fund; 1998.

5. National Center for Injury Prevention and Control, Center for Disease Control and Prevention. Costs of Intimate Partner Violence Against Women in the United States. Atlanta, GA: National Center for Injury Prevention and Control, Center for Disease Control and Prevention; 2003.

6. Tjaden P, Thoennes N. Extent, Nature, and Consequences of Intimate Partner Violence: Findings from the National Violence Against Women Survey. Washington, DC: Department of Justice; 2000.

7. Grana SJ. Sociostructural considerations of domestic femicide. Fam Violence. 2001;16:421-35.

8. Centers for Disease Control. Prevalence of intimate partner violence and injuries-Washington, 1998. MMWR. 2000;49:589-92.

9. Tjaden P, Thoennes N. Full Report of the Prevalence, Incidence, and Consequences of Violence against Women: Research Report. Washington, DC: Department of Justice; 2000.

10. Saltzman LE, Green YT, Marks JS, Thacker SB. Violence against women as a public health issue: comments from the CDC. Am J Prev Med. 2000; 19:325-9.

11. Kernic MA, Wolf ME, Holt VL. Rates and relative risk of hospital admission among women in violent intimate partner relationships. Am J Public Health. 2000;90:1416-20.

12. Campbell JC. Health consequences of intimate partner violence. Lancet. 2002;359:1331-6.

13. Silverman JG, Raj A, Mucci LA, Hathaway JE. Dating violence against adolescent girls and associated substance use, unhealthy weight control, sexual risk behavior, pregnancy, and suicidality. JAMA. 2001;286: 572-9.

14. Eisenstat SA, Bancroft L. Domestic violence. N Engl J Med. 1999;341: 886-92.

15. Campbell JC, Lewandowski LA. Mental and physical health effects of intimate partner violence on women and children. Psychiatr Clin North Am. 1997;20:353-74.

16. Fogarty CT, Burge S, McCord EC. Communicating with patients about intimate partner violence: screening and interviewing approaches. Fam Med. 2002;34:369-75.

17. Rodriguez MA, Sheldon WR, Bauer HM, Perez-Stable EJ. The factors associated with disclosure of intimate partner abuse to clinicians. J Fam Pract. 2001;50:338-44.

18. Caralis PV, Musialowski R. Women's experiences with domestic violence and their attitudes and expectations regarding medical care of abuse victims. South Med J. 1997;90:1075-80.

19. Titus $\mathbf{K}$. When physicians ask, women tell about domestic abuse and violence. JAMA. 1996;275:1863-5.

20. Elliott L, Nerney M, Jones T, Friedmann PD. Barriers to screening for domestic violence. J Gen Intern Med. 2002;17:112-6.

21. Rodriguez MA, Bauer HM, McLoughlin E, Grumbach K. Screening and intervention for intimate partner abuse: practices and attitudes of primary care physicians. JAMA. 1999;282:468-74.

22. Waalen J, Goodwin MM, Spitz AM, Petersen R, Saltzman LE. Screening for intimate partner violence by health care providers. Barriers and interventions. Am J Prev Med. 2000; 19:230-7.

23. Schulman KA, Berlin JA, Harless W, et al. The effect of race and sex on physicians' recommendations for cardiac catheterization. N Engl J Med. 1999;340:618-26.

24. Smedley BD, Stith AY, Nelson AR, eds. Institute of Medicine Committee on Understanding and Eliminating Racial and Ethnic Disparities in Health Care, Board on Health Sciences Policy. Unequal Treatment: Confronting Racial and Ethnic Disparities in Health Care. Washington, DC: National Academy Press; 2003.

25. Recommendations for Clinical Preventive Services. U.S. Preventive Services Task Force. Available at: www.ahrq.gov/clinic/uspstfix. htm\#Recommendatins. Accessed July 28, 2004.

26. Nelson HD, Nygren P, McInerney Y, Klein J. U.S. preventive services task force. Screening women and elderly adults for family and intimate partner violence: a review of the evidence for the US Preventive Services Task Force. Ann Intern Med. 2004; 140:387-96.

27. van Ryn M, Burke $J$. The effect of patient race and socio-economic status on physicians' perceptions of patients. Soc Sci Med. 2000;50:813-28. 
28. van Ryn M. Research on the provider contribution to race/ethnicity disparities in medical care. Med Care. 2002;40(suppl 1):I140-51.

29. Rose GL, Rukstalis MR, Schuckit MA. Informal mentoring between faculty and medical students. Acad Med. 2005;80:344-8.

30. Alexander GC, Casalino LP, Meltzer DO. Patient-physician communication about out-of-pocket costs. JAMA. 2003;290:953-8.

31. Brienza RS, Whitman L, Ladouceur L, Green ML. Evaluation of a women's safe shelter experience to teach internal medicine residents about intimate partner violence. A randomized controlled trial. J Gen Intern Med. 2005;20:536-40.

32. McCauley J, Jenckes MW, McNutt LA. ASSERT: the effectiveness of a continuing medical education video on knowledge and attitudes about interpersonal violence. Acad Med. 2003;78:518-24.

33. Nicolaidis C, Curry M, Gerrity M. Measuring the impact of the voices of survivors program on health care workers' attitudes toward survivors of intimate partner violence. J Gen Intern Med. 2005;20:731-7.

34. Sugg NK, Inui T. Primary care physicians' response to domestic violence. Opening pandora's box. JAMA. 1992;267:3157-60.

\section{Supplementary Material}

The following supplementary material is available for this article online at www.blackwell-synergy.com

\section{Appendix}

35. Borowsky IW, Ireland M. Parental screening for intimate partner violence by pediatricians and family physicians. Pediatrics. 2002;110:50916.

36. Hayden SR, Barton ED, Hayden M. Domestic violence in the emergency department: how do women prefer to disclose and discuss the issues? J Emerg Med. 1997;15:447-51.

37. Tatara T, Kuzmeskus-Blumerman L, Duckhorn E, et al. The National Elder Abuse Incidence Study (NEAIS); Final report. National Center on Elder Abuse at the American Public Human Services Association in Collaboration with Westat Inc. for the Administration for Children and Families and the Administration on Aging in the U.S. Department of Health and Human Services; 1998.

38. Friedman LS, Samet JH, Roberts MS, Hudlin M, Hans P. Inquiry about victimization experiences. A survey of patient preferences and physcian practices. Arch Intern Med. 1992;152:1186-90.

39. Asch DA, Jedrziewski MK, Christakis NA. Response rates to mail surveys published in medical journals. J Clin Epidemiol. 1997;50:1129-36. 